Submission ID: 43799

\title{
Atlas of the Multiphysical Models of Westen Siberia Reservoir
}

G.V. Nesterova* (IPGG SB RAS), I.N. Yeltsov (IPGG SB RAS), A.Y. Sobolev (IPGG SB RAS), I.V. Surodina (ICMMG SB RAS)

\section{SUMMARY}

ATLAS is the collection of multiphysical models of the typical reservoirs of Western Siberia. Both geomechanical, hydrodynamic, geoelectric processes in the vicinity of the borehole and electrical and electromagnetic well logs are jointly analyzed. The influence of each of the hydrophysical and geomechanical parameters on the process of mud filtrate invasion into the formation, evolution of the electrical resistivity distribution in the borehole environment, the electric and electromagnetic logs can be studied and visualised by using ATLAS.

Further development of ATLAS is aimed to creating interpreting system of the well geophysical and geological-technological studies on the basis of multiphysical reservoir models. 


\section{Атлас синтетических многофизичных моделей коллекторов Западной Сибири}

Г.В. Нестерова* (ИНГГ СО РАН), И.Н. Ельцов (ИНГГ СО РАН), А.Ю. Соболев (ИНГГ СО РАН), И.В. Суродина (ИВМиМГ СО РАН)

\section{Введение}

Разработаны методика и программно-алгоритмическое обеспечение для создания многофизичных моделей пластов-коллекторов, включающих описание геомеханических, гидродинамических и геоэлектрических процессов в окрестности скважины (Ельцов и др., 2012; Eltsov et al., 2013; Ельцов и др., 2014; Нестерова и др., 2014; GEHM2, 2017). Применение моделей для решения задачи оценки пласта на основе комплексной интерпретации данных геофизических (ГИС) и геолого-технологических (ГТИ) исследований скважины осложняется большим количеством (около 30) входных параметров многофизичной модели и достаточно большим временем расчёта. Целью этой работы было сформировать систематизированный набор (АТЛАС) синтетических многофизичных моделей типичных для Западной Сибири коллекторов (ATLAS_GEHM, 2017), пользуясь которым можно было бы изучать влияние каждого из параметров единой модели на формирование зоны проникновения, эволюцию геоэлектрической модели пласта, прогнозировать показания приборов электрического и электромагнитного каротажа. Каждый элемент АТЛАСА, которым является единая электрогидродинамическая и геомеханическая (ЭГДиГМ) модель пласта, включает: параметрическое описание коллектора, бурового раствора, режима бурения скважины; рассчитанные поля давления, водонасыщенности, солёности, удельного электрического сопротивления (УЭС), синтетические (рассчитанные по УЭС) показания приборов электрического и электромагнитного каротажа (Рисунок 1).

\section{Методы}

Большинство современных совместных флюидо-геомеханических моделей реализуют блочно-модульные подходы для моделирования проникновения бурового раствора в коллектор и напряжённо-деформированного состояния, а также различные схемы согласования модулей (coupling) (Thomas et al., 2003; Tran et al. 2005; Settari and Sen, 2008; Moinfar et al., 2013; Lautenschläger et al., 2013). Такой же подход использован авторами методики построения ЭГДиГМ моделей пластов (Yeltsov et al., 2015; Ельцов и др., 2017): для описания процесса проникновения бурового раствора в продуктивный пласт схема включает геомеханическую (поропластическая модель с условием Треска или Кулона-Мора) и гидродинамическую (модель Бакли-Леверетта двухфазной фильтрации) составляющие. Для расчёта УЭС по гидродинамическим параметрам используется одна из формул электропроводности гетерогенных сред (Archie, 1942; Bussian, 1983; Lima and Sharma, 1990; Sheng, 1991; Глинских и др., 2014). В ЭГДиГМ модели учитывается эмпирическая зависимость проницаемости от напряжений, построенная на основе экспериментальных данных (Dobrynin, 1962; Holt, 1990; Григорьев, Шубин, 2015), один из параметров которой - отношение минимального (вблизи скважины) и максимального (в нетронутой части пласта) значений проницаемости приведен в таблице 1.

Таблица 1. Основные параметры ЭГДиГМ модели

\begin{tabular}{|l|c|l|c|}
\hline \multicolumn{1}{|c|}{ Параметр } & $\begin{array}{l}\text { Диапазон } \\
\text { значений } \\
\text { параметров }\end{array}$ & Параметр & $\begin{array}{l}\text { Диапазон } \\
\text { значений } \\
\text { параметров }\end{array}$ \\
\hline Глубина, Depth, м & $2500-4000$ & $\begin{array}{l}\text { Содержание твёрдых частиц } \\
\text { в буровом растворе, del, \% }\end{array}$ & $4-5$ \\
\hline Пористость, Por,\% & $5-25$ & $\begin{array}{l}\text { Репрессия в \% от пластового } \\
\text { давления, zb }\end{array}$ & $5-10$ \\
\hline Проницаемость, Per, мД & $1-200$ & $\begin{array}{l}\text { Прочность образца на } \\
\text { сжатие в пластовых }\end{array}$ & $9-15$ \\
\hline
\end{tabular}




\begin{tabular}{|c|c|c|c|}
\hline & & условиях, Ts, МПа & \\
\hline $\begin{array}{l}\text { Нефтенасыщенность, (1- } \\
\text { Sw), \% }\end{array}$ & $50-90$ & $\begin{array}{l}\text { Угол внутреннего трения } \\
\text { пород скелета коллектора, } \\
\text { alf, град }\end{array}$ & $15-25$ \\
\hline $\begin{array}{l}\text { Отношение вязкостей } \\
\text { пластовых воды и нефти, } \\
\text { mu_w_o }\end{array}$ & $0.1-0.5$ & $\begin{array}{l}\text { Максимальный коэффициент } \\
\text { бокового распора, } \mathrm{Q}_{\mathrm{y}}\end{array}$ & $0.7-0.8$ \\
\hline $\begin{array}{l}\text { Минерализация пластовых } \\
\text { вод, Cw0, г/л }\end{array}$ & $6-32$ & $\begin{array}{l}\text { Минимальный коэффициент } \\
\text { бокового распора, } \mathrm{Q}_{x}\end{array}$ & $0.61-0.8$ \\
\hline $\begin{array}{l}\text { Минерализация бурового } \\
\text { раствора, Cw1, г/л }\end{array}$ & $1-10$ & $\begin{array}{l}\text { Отношение минимального и } \\
\text { максимального значений } \\
\text { проницаемости, mu_w_o }\end{array}$ & $0.2-1.0$ \\
\hline
\end{tabular}

Особое внимание уделено изучению влияния аксиальной неоднородности распределения проницаемости на каротажные диаграммы прибора ВИКИЗ и БКЗ. Алгоритмы расчёта таких диаграмм для супермощных компьютеров с параллельными процессорами разработаны И.В. Суродиной (Суродина и Нестерова, 2015), расчёты проводились в Сибирском Суперкомпьютерном Центре (http://www2.sscc.ru/).
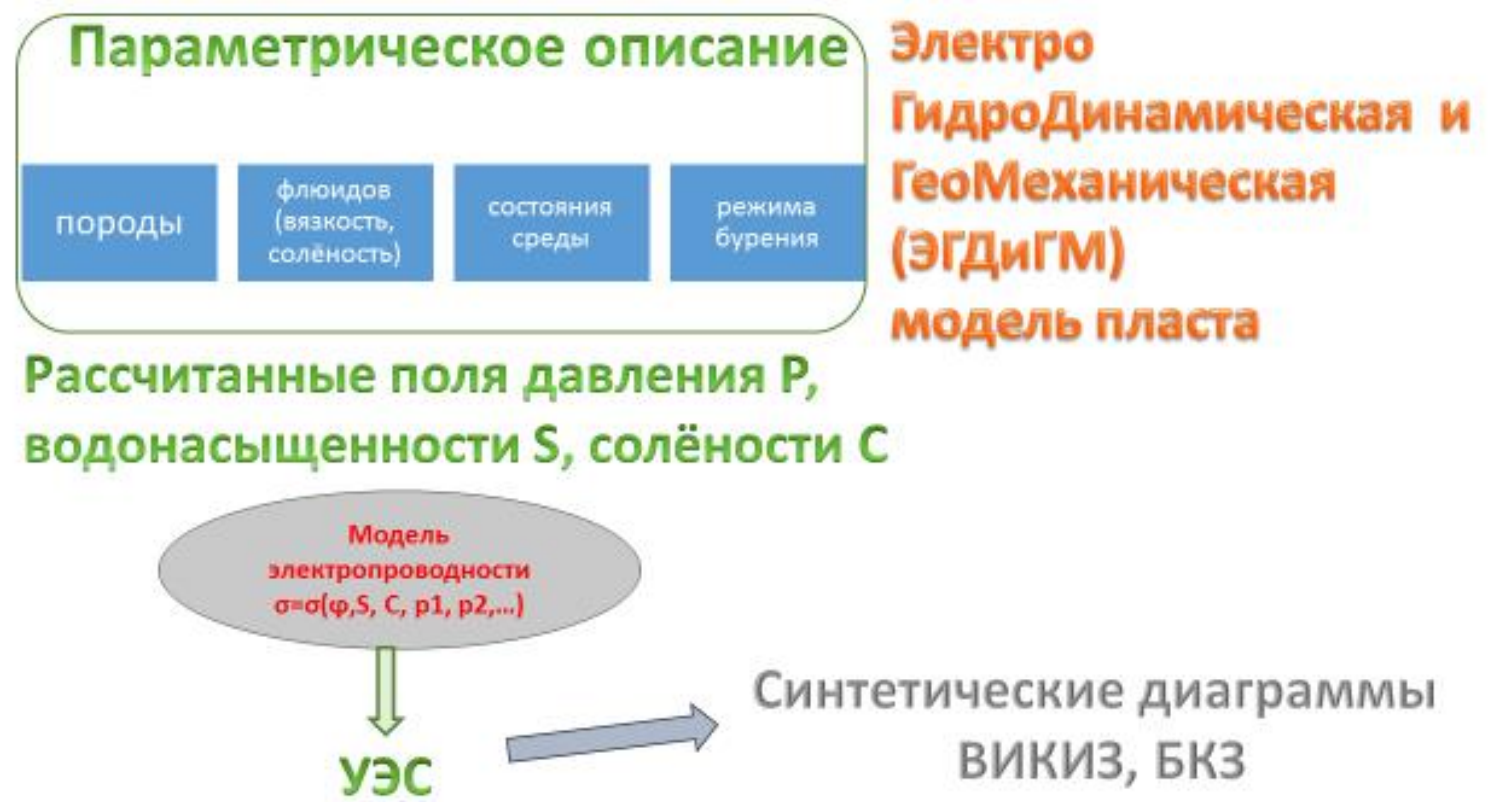

\section{Рисунок 1. Схема ЭГДиГМ модели пласта}

Параметры ЭГДиГМ модели и рассчитанные синтетические сигналы сохраняются в базе данных вместе со ссылками на архивы рассчитанных полей, которые из-за большого объема располагаются в файловом хранилище. Клиентское приложение предоставляет доступ к этой базе и позволяет выбирать модели с заданными ограничениями на диапазоны параметров. На рисунке 2 представлен фрагмент входных параметров ЭГДиГМ модели, яркой чёрной линией выделена выбранная к просмотру (изучению) модель. Визуализация многомерных данных методом параллельных координат использует реализацию d3.parcoords.js (Chang, 2012-2017) с помощью библиотеки D3 (Bostock et al., 2011; https://d3js.org). 


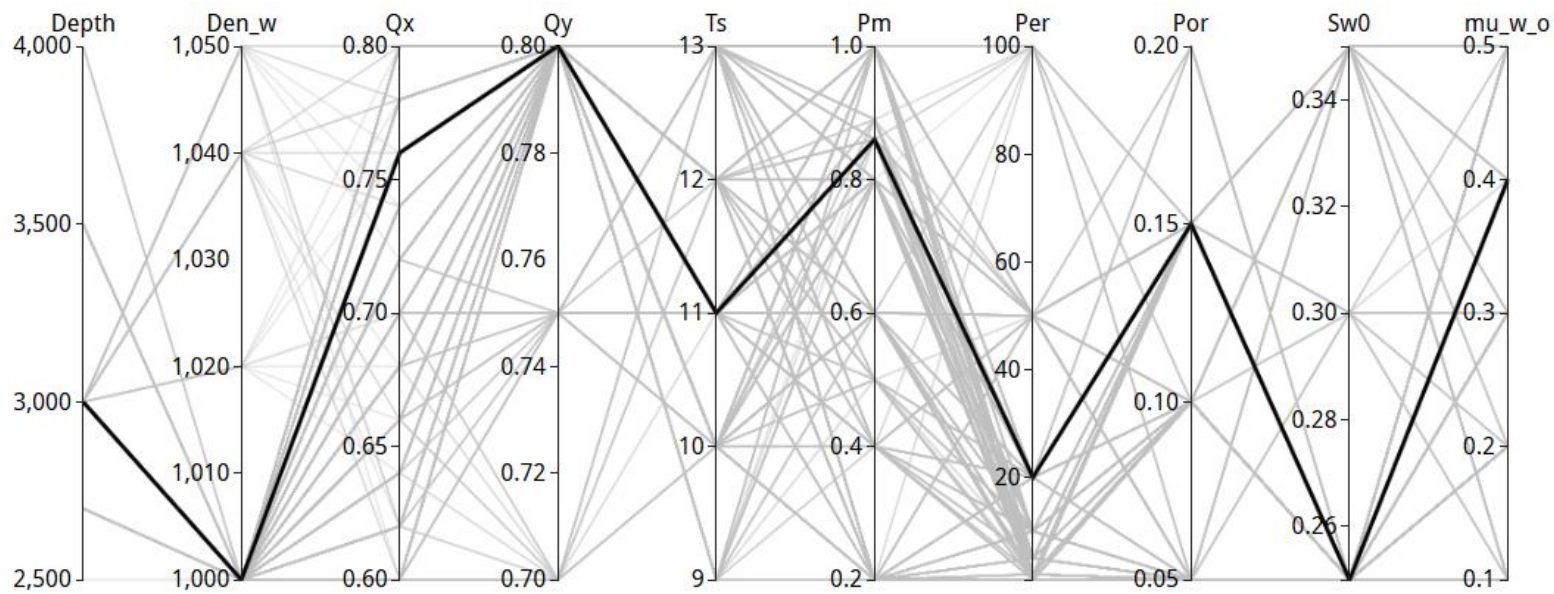

Рисунок 2. Представление параметров модели методом параллельных координат. Обозначения параметров приведены в таблице 1

\section{Выводы}

Предлагаемый АТЛАС позволяет получить представление об особенностях формирования зоны проникновения, эволюции её свойств и физических полей для применяемых на практике технологий бурения и исследования скважин. Расчёты показывают, что в типичных условиях бурения терригенных разрезов необходимо учитывать и геомеханические и гидродинамические условия формирования прискважинной зоны, только тогда можно эффективно интерпретировать каротажные данные и достоверно оценивать фильтрационно-емкостные параметры пласта. Дальнейшее развитие АТЛАСА направлено на создание на его базе инструментария для интерпретации комплекса данных ГИС и ГТИ на основе многофизичных моделей пластов-коллекторов.

Работа выполнена при поддержке гранта РФФИ 16-05-00830.

\section{Библиография}

1. Глинских, В.Н., Нестерова, Г.В., Эпов, М.И. [2014] Моделирование и инверсия данных электромагнитного каротажа с использованием петрофизических моделей электропроводности. Геология и геофизика, 55 (5-6), 1001-1010.

2. Григорьев, Б.В., Шубин, А.А. [2015] Влияние изменения эффективного давления на фильтрационно-емкостные свойства пород-коллекторов. Вестник Тюменского гос.университета. Физико-математическое моделирование. Нефть, газ, энергетика, 1 (2). $15-21$.

3. Ельцов, И.Н., Назаров, .А., Назарова, Л.А., Нестерова, .В., Эпов, М.И. [2012] Интерпретация геофизических измерений в скважинах с учетом гидродинамических и геомеханических процессов в зоне проникновения. ДАН, 445 (6), 671-674.

4. Ельцов, И.Н., Назарова, Л.А., Назаров, Л.А., Нестерова, Г.В., Соболев, А.Ю., Эпов М.И. [2014] Скважинная геоэлектрика нефтегазовых пластов, разбуриваемых на репрессии давления в неравнокомпонентном поле напряжений. Геология и геофизика, 55 (56), 978-990.

5. Ельцов И.Н., Нестерова Г.В., Назаров Л.А., Назарова Л.А. [2017] Совместные электрофизические и геомеханические модели нефтенасыщенных коллекторов. 
Тюмень 2017 «Геонауки - ключ к рациональному освоению недр», 5-я научнопрактическая конференция, 27-30 марта, электронный ресурс.

6. Нестерова, Г.В., Ельцов, И.Н., Киндюк, В.А., Назаров, Л.А., Назарова, Л.А. [2014] Моделирование гидродинамических процессов в напряжённо-деформированной прискважинной зоне и геофизические приложения. Петрофизика сложных коллекторов: проблемы и перспективы 2014, Сборник статей, М.: «ЕАГЕ Геомодель», 327-344.

7. GEHM2. Свидетельство о государственной регистрации программ для ЭВМ / Назаров, Л.А., Назарова, Л.А., Нестерова, Г.В., Ельцов, И.Н. // Правообладатель: Институт нефтегазовой геологии и геофизики им. А.А. Трофимука СО РАН. - Свид-во о прогр. № 2017619772; заявл. 14.12.2016, опубл. 19.04.2017.

8. ATLAS_GEHM. Свидетельство о государственной регистрации базы данных / Ельцов, И.Н., Назаров, Л.А., Назарова, Л.А., Нестерова, Г.В., Соболев, А.Ю. // Правообладатель: Институт нефтегазовой геологии и геофизики им. А.А. Трофимука СО РАН. - Свид-во о БД № 2017619764; заявл. 14.12.2016, опубл. 18.04.2017.

9. Суродина, И.В, Нестерова, Г.В. [2015] Моделирование показаний зондов ВИКИЗ и БКЗ на графических процессорах. Петрофизика сложных коллекторов: проблемы и перспективы 2015. Сборник статей EAGE, 85-94.

10. Archie, G.E. [1942] The electrical resistivity $\log$ as an aid in determining some reservoir characteristics, Transactions of AIME, 146, 54-62.

11. Bostock, M., Ogievetsky V. and Heer J. [2011] $\mathrm{D}^{3}$ data-driven documents: IEEE transactions on visualization and computer graphics, 12, 2301-2309.

12. Bussian, A.E. [1983] Electrical conductance in a porous medium, Geophysics, 48, 12581268.

13. Chang, K. [2012-2017] Parallel Coordinates. A visual toolkit for multidimensional detectives: URL: http://syntagmatic.github.io/parallel-coordinates.

14. Dobrynin, V.M. [1962] Effect of overburden pressure on some properties of sandstones: SPE journal, 12, 360-366.

15. Eltsov, I., Kuvshinov, V., Nasarov, L., Nazarova, L., Nesterova, G., Stefanov, Y. and Shelukhin V. [2013] Multiphysics model of borehole environment and joint inversion of well logs: SAGA 13th biennial conference and 6th International AEM, Abstract, 55-56.

16. Holt, R.M. [1990] Permeability reduction induced by a mon hydrostatic stress field. Formation Evaluation: SPE, 12, 444-448.

17. Lautenschläger, C.E.R., Righetto, G.L., Inoue, N. and Barreto da Fontoura, S.A. [2013] Advances on partial coupling in reservoir simulation: A new scheme of hydromechanical coupling: North Africa Technical Conference \& Exhibition, Conference Paper 164657-MS. $8 \mathrm{p}$.

18. Lima, de O.A.L. and Sharma, M.M. [1990] A grain conductivity approach to shaly sandstones, Geophysics 55 (10), 1347-1356.

19. Moinfar, K., Johns, R.T. and Varavei, A. [2013] Coupled Geomechanics and Flow Simulation for an Embedded Discrete Fracture Model. SPE Reservoir Simulation Symposium, 18-20 February, The Woodlands, Texas, USA, SPE-163666-MS, https://doi.org/10.2118/163666-MS.

20. Sheng, P. [1991] Consistent modeling of the electrical and elastic properties of sedimentary rocks, Geophysics, 8, 1236-1243.

21. Settari, A.T. and Sen, V. [2008] Geomechanics in Integrated Reservoir Modeling: Offshore Technology Conference held in Houston, Texas, Conference paper 19530-MS, 9 p.

22. Thomas, L.K., Chin, L.Y., Pierson, R.G. and Sylte, J.E. [2003] Coupled Geomechanics and reservoir simulation: SPE Journal, 12, 350-358.

23. Tran, D., Nghiem, L. and Buchanan, L. [2005] Iterative coupling of geomechanics with reservoir simulation: SPE Reservoir Simulation Symposium, Conference paper 93244-MS, 8 p.

24. Yeltsov, I., Nesterova, G., Nazarova, L. and Nazarov, L. [2015] Multidisciplinary model of borehole environment and formation evaluation: The World Multidisciplinary Earth Sciences Symposium - WMESS 2015 Abstract Collection, 318-318. 


\section{References}

1. Glinskikh, V.N., Nesterova, G.V., Epov M.I. [2014] Forward modeling and inversion of induction logs from shaly sand reservoirs using petrophysical conductivity models. Russian Geology and Geophysics, 55 (5-6), 793-799.

2. Grigoriev B.V., Shubin, A.A. [2015] Impact of effective pressure modification on filtrationvolumetric characterisnics of reservoir rocks. Bulletin of Tyumen University: physical and mathematical modeling. Oil, Gas, Energetics, 1 (2). 15-21.

3. Yeltsov, I.N., Nesterova, G.V., Nazarov, L.A., Nazarova, L.A., Epov, M.I. [2012] Logging interpretation taking into accounthydrodynamical and geomechanical processes in an invaded zone. Doklady Earth Science, 445 (2), 1021-1024.

4. Yeltsov, I.N., Nesterova, G.V., Sobolev, A.Y., Epov, M.I., Nazarova, L.A., Nazarov, L.A. [2014] Geomechanics and fluid flow effects on electric well logs: multiphysics modeling. Russian Geology and Geophysics, 55 (5-6), 775-783.

5. Yeltsov, I.N., Nesterova, G.V., Nazarov, L.A., Nazarova, L.A. [2017] Coupled electrophysical and geomechanical models of oil-saturated reservoirs. Abstracts of $5^{\text {th }}$ Scientific-Practical Conference "Tyumen 2017". DOI: 10.3997/2214-4609.201700144.

6. Nesterova, G.V., Yeltsov, I.N., Kindyuk, V.A., Nazarov, L.A., Nazarova, L.A. [2014] Simulation of hydrodynamic processes in the borehole environment under stress-strain state and geophysical applications. EAGE collection of articles "Petrophysics of complicated reservoirs: Problems and Perspectives 2014", 327-344.

7. GEHM2. Certificate of state registration of computer program / Nazarov L.A., Nazarova L.A,Yeltsov I.N., Nesterova G.V. // Right holder: Trofimuk Institute of Petroleum Geology and Geophysics SB RAS. Certificate № 2017619772; publ. 19.04.2017.

8. ATLAS_GEHM. Certificate of state registration of database / Yeltsov I.N., Nazarov L.A., Nazarova L.A. Nesterova G.V., Sobolev A.Y. // Right holder: Trofimuk Institute of Petroleum Geology and Geophysics SB RAS. Certificate № 2017619764; publ. 18.04.2017.

9. Surodina I.V., Nesterova G.V. [2015] VIKIZ and BKZ log simulation using Graphics Processing Units. EAGE collection of articles "Petrophysics of complicated reservoirs: Problems and Perspectives 2015", 85-94.

10. Archie, G.E. [1942] The electrical resistivity $\log$ as an aid in determining some reservoir characteristics, Transactions of AIME, 146, 54-62.

11. Bostock, M., Ogievetsky V. and Heer J. [2011] D $\mathrm{D}^{3}$ data-driven documents: IEEE transactions on visualization and computer graphics, 12, 2301-2309.

12. Bussian, A.E. [1983] Electrical conductance in a porous medium, Geophysics, 48, 12581268.

13. Chang, K. [2012-2017] Parallel Coordinates. A visual toolkit for multidimensional detectives: URL: http://syntagmatic.github.io/parallel-coordinates.

14. Dobrynin, V.M. [1962] Effect of overburden pressure on some properties of sandstones: SPE journal, 12, 360-366.

15. Eltsov, I., Kuvshinov, V., Nasarov, L., Nazarova, L., Nesterova, G., Stefanov, Y. and Shelukhin V. [2013] Multiphysics model of borehole environment and joint inversion of well logs: SAGA 13th biennial conference and 6th International AEM, Abstract, 55-56.

16. Holt, R.M. [1990] Permeability reduction induced by a mon hydrostatic stress field. Formation Evaluation: SPE, 12, 444-448.

17. Lautenschläger, C.E.R., Righetto, G.L., Inoue, N. and Barreto da Fontoura, S.A. [2013] Advances on partial coupling in reservoir simulation: A new scheme of hydromechanical coupling: North Africa Technical Conference \& Exhibition, Conference Paper 164657-MS. $8 \mathrm{p}$.

18. Lima, de O.A.L. and Sharma, M.M. [1990] A grain conductivity approach to shaly sandstones, Geophysics 55 (10), 1347-1356.

19. Moinfar, K., Johns, R.T. and Varavei, A. [2013] Coupled Geomechanics and Flow Simulation for an Embedded Discrete Fracture Model. SPE Reservoir Simulation Symposium, 18-20 February, The Woodlands, Texas, USA, SPE-163666-MS, https://doi.org/10.2118/163666-MS. 
20. Sheng, P. [1991] Consistent modeling of the electrical and elastic properties of sedimentary rocks, Geophysics, 8, 1236-1243.

21. Settari, A.T. and Sen, V. [2008] Geomechanics in Integrated Reservoir Modeling: Offshore Technology Conference held in Houston, Texas, Conference paper 19530-MS, 9 p.

22. Thomas, L.K., Chin, L.Y., Pierson, R.G. and Sylte, J.E. [2003] Coupled Geomechanics and reservoir simulation: SPE Journal, 12, 350-358.

23. Tran, D., Nghiem, L. and Buchanan, L. [2005] Iterative coupling of geomechanics with reservoir simulation: SPE Reservoir Simulation Symposium, Conference paper 93244-MS, 8 p.

24. Yeltsov, I., Nesterova, G., Nazarova, L. and Nazarov, L. [2015] Multidisciplinary model of borehole environment and formation evaluation: The World Multidisciplinary Earth Sciences Symposium - WMESS 2015 Abstract Collection, 318-318. 